\title{
MONITORAMENTO DE PACIENTES HOMECARE: UMA ABORDAGEM UTILIZANDO BANCO DE DADOS EM TEMPO REAL
}

\author{
Yáskara Ygara Menescal Pinto Fernandes \\ Mestre pelo Programa de Pós-Graduação em Engenharia Elétrica, da Universidade Federal \\ de Campina Grande, 2005. Atualmente é Professora da Universidade Federal Rural do \\ Semiárido. Email: yaskaramenescal@ufersa.edu.br

\section{Cicília Raquel Maia Leite}

Mestrado em Engenharia Elétrica pela Universidade Federal de Campina Grande, 2005. Doutorado em Engenharia Elétrica e de Computação pela Universidade Federal do Rio Grande do Norte, 2011. Pós-doutorado no Massachusetts Institute of Technology (MIT) no Laboratory of Computational Physiology, Harvard-MIT Division of Health Science and Technology. Professora da UERN, Adjunto IV, no Departamento de Informática. Professora Permanente do Programa de Pós-Graduação em Ciência da Computação Associação Ampla UERN/UFERSA. Email: ciciliamaia@gmail.com

\section{Ricardo Alexandro de Medeiros Valentim}

Doutor em Engenharia Elétrica e de Computação, Coordenador do Laboratório de Inovação Tecnológica em Saúde do Hospital Universitário Onofre Lopes, Professor do Departamento de Engenharia Biomédica da Universidade Federal do Rio Grande do Norte.

\section{E-mail: ricardo.valentim@ufrnet.br}

\section{Helio Roberto Hekis}

Doutor em Engenharia de Produção e Sistemas pela Universidade Federal de Santa Catarina - UFSC (2004). Pesquisador do Laboratório de Inovação Tecnológica em Saúde (LAIS) - Hospital Universitário Onofre Lopes (HUOL). Professor do Departamento de

Engenharia de Produção - Universidade Federal do Rio Grande do Norte (UFRN). hekis1963@gmail.com

Robinson Luís de Souza Alves

Laboratório de Inovação Tecnológica em Saúde (LAIS) - Hospital Universitário Onofre Lopes (HUOL). Instituto Federal de Educação, Ciência e Tecnologia do Rio Grande do Norte (IFRN). robinson.alves@ig.com.br

Idelmárcia Dantas de Oliveira

Funcionária e Mestra em Engenharia de Produção pela Universidade Federal do Rio Grande do Norte (UFRN). idelmarcia@gmail.com

\section{RESUMO}

Ultimamente a quantidade de pessoas que precisam de atendimento em hospitais públicos e privados cresce devido, entre outros fatores, ao aumento da população mundial e a demora no atendimento de pacientes. Este é um problema sério enfrentado por administradores de hospitais que a cada dia está mais difícil de oferecer um serviço de qualidade a quem os procura. Uma das formas de melhorar a qualidade do atendimento do paciente pode ser através do desenvolvimento de sistemas de homecare que possibilitam que o paciente receba um tratamento clínico de qualidade diretamente em sua residência. No entanto, o ambiente domiciliar pode se tornar complexo, visto que muitas vezes o cuidador é alguém da própria família e não possui a correta técnica de monitoramento ou está desatento em função do desgaste provocado pelo intenso cuidado ao paciente. Assim, torna-se 
importante o desenvolvimento de soluções que diminuam a sobrecarga do cuidador, ao passo em que o paciente continue sendo monitorado. Uma maneira de alcançar tais soluções é através da aplicação de conceitos de computação pervasiva em ambientes domiciliares. Dessa forma, o ambiente é povoado de sensores e outros dispositivos computacionais, onde o paciente pode ser monitorado constantemente e o ambiente pode se adaptar às necessidades deste usuário, antecipando-se a situações de riscos de acordo com o contexto atual. Assim, a arquitetura proposta utiliza conceitos de computação pervasiva possibilitando acesso à informação a qualquer hora e independente do lugar onde o paciente esteja, uma vez que um ambiente homecare possui um alto grau de dinamicidade dos envolvidos. Para o tratamento dos dados que serão coletados pelos sensores é preciso de uma tecnologia que execute os dados em tempo real, ou seja, em tempo hábil para que seja feita a tomada de decisão pelos profissionais de saúde. Dessa forma, utiliza-se um banco de dados em tempo real para tratar dados e transações em tempo real garantindo a consistência lógica e temporal dos mesmos, além de gerenciar grandes volumes de dados. Assim, a especificação do sistema foi modelada em UML e consequentemente a definição da arquitetura.

PALAVRAS-CHAVE: Sistemas homecare, computação pervasiva, banco de dados em tempo-real.

\title{
MONITORING OF PATIENTS HOMECARE: AN APPROACH USING REAL- TIME DATABASE
}

\begin{abstract}
Lately the amount of people who need care in public and private hospitals is growing due, among other factors, the increase of the world population and the delay in the treatment of patients. This is a serious problem faced by administrators of hospitals that each day is more difficult to provide a quality service to those who demand. One of the ways to improve the quality of patient care may be through the development of systems of homecare that empowers the patient receives a clinical treatment of quality directly into their residence. However, the home environment can become complex, since many times the caregiver is someone's own family and doesn't have the correct technique of monitoring or is inattentive in function of the wear caused by intense care to the patient. Thus, it is important for the development of solutions that reduce the overhead of the caregiver, the step in which the patient continue being monitored. One way to achieve such solutions is through the application of concepts of pervasive computing environments at home. In this way, the environment is only populated for sensors and other computing devices, where the patient can be monitored constantly and the environment can adapt to the needs of this user, anticipating to situations of risk according to the current context. Thus, the proposed architecture uses concepts of pervasive computing by providing access to information at any time and regardless of the place where the patient is, once an environment homecare has a high degree of dynamics of the people involved. For the treatment of the data that will be collected by the sensors is need of a technology that perform the data in real time, id est, in a timely manner so that it made the decision-making by health professionals. In this way, it uses a real-time database to deal with data and transactions in real time while ensuring the logical consistency and temporal of same, in addition to managing large volumes of data. Thus, the specification of the system was modeled in UML and consequently the definition.
\end{abstract}

KEYWORDS: homecare system, pervasive computing, database in real-time. 


\section{MONITORAMENTO DE PACIENTES HOMECARE: UMA ABORDAGEM UTILIZANDO BANCO DE DADOS EM TEMPO REAL}

\section{INTRODUÇÃ̃}

Atualmente, um dos principais problemas enfrentados por administradores de hospitais é oferecer a garantia de um serviço de qualidade a seus clientes. Nos últimos anos, os hospitais públicos e privados vêm aumentando a demanda de serviços por diversos fatores como, por exemplo, alta demanda de pacientes, problemas de infra-estrutura, comunicação interna falha que pode compromete o tratamento clínico do paciente (Andrade et al., 2009).

Uma das formas de minimizar a demora no atendimento é através do desenvolvimento de aplicações computacionais que agilizem o fluxo de tratamento de pacientes. A grande maioria dos hospitais têm sistemas informatizados para gerenciar o trabalho burocrático e administrativo, como também, possuem aplicações que tornam o trabalho dos clínicos mais otimizados. No entanto, esses sistemas formam projetados para serem utilizados em locais fixos, onde o usuário deve utilizar o computador em suas salas (Zarghami, et al., 2011). Dessa forma, este tipo de aplicação não pode ser considerado como ideal, uma vez que os hospitais exigem um alto grau de mobilidade dos profissionais e um trabalho colaborativo entre especialistas de diferentes áreas, além de ser um ambiente propício para ocorrer frequentes interrupções nas atividades que estes profissionais realizam.

Uma alternativa de solucionar os problemas enfrentados em um hospital é o paciente receber serviços clínicos em suas residências, através da implantação de aplicações de homecare. Um ambiente homecare é caracterizado por possibilitar que o paciente receba atendimento médico em sua residência, seja por necessidade, como em casos onde o paciente é impossibilitado de locomoção, ou até por vontade própria, uma vez que o paciente ficará mais tempo junto de seus familiares e amigos. Assim, pode torna-se bastante dinâmico, uma vez que o paciente pode locomove-se em sua residência a qualquer momento ou ainda receber visitas de parentes e amigos. No entanto, o paciente deve está sendo monitorado continuamente, e qualquer anormalidade identificada deve ser tratada imediatamente.

A quantidade de pessoas idosas, em especial aquelas com doenças crônicas ou condições críticas de saúde, tem crescido substancialmente nos últimos anos. Este cenário provoca uma alta demanda, exigindo cada vez mais da atual infraestrutura de serviços de saúde. A assistência domiciliar a saúde com o uso da tecnologia de computação pervasiva, pode representar uma solução (Copetti, 2010).

Dessa forma, a computação pervasiva apresenta característica que possibilita a interação entre os dispositivos e usuários de forma mais intuitiva possível, ou seja, o paciente realiza uma determinada tarefa sem perceber que está utilizando o computador para tal (Nogueira et al.; 2011). Em um ambiente homecare pervasivo, os profissionais da saúde tem acesso rápido e seguro as informações dos pacientes mesmo não encontrando em seu local de trabalho. Assim, é possível a tomada de decisões mais rapidamente sobre o estado de saúde do paciente. No entanto, os dados que serão coletados durante o monitoramento do 
paciente devem ser tratados e processados em tempo hábil para que o paciente não sofra nenhuma sequela.

Assim, Bancos de Dados em Tempo Real (BDTR) tem-se consolidado como uma área de pesquisa em expansão, para tratar com processamento de informações que geram grandes volumes de dados e que apresentem um tempo de vida útil limitado (informação com prazo de validade). Esses sistemas possuem a importância de utilizar dados que, quando avaliados de forma on-line tem uma validade temporal que deve ser respeitada pelo sistema, como forma de garantir a sua consistência lógica e temporal (Shanker et al.,2008).

Portanto, a importância de utilizar BDTR para monitoramento de pacientes, que tem como apresentem doenças crônicas e que necessitam serem tratados em suas residências, possibilitando uma comunicação constante entre a residência do paciente e o hospital que está oferecendo serviço e a equipe médica.

O artigo está organizado da seguinte forma: na Seção 2 são apresentados os conceitos de assistência domiciliar a saúde e sistemas homecare, bem como a descrição das características desses sistemas; na Seção 3 são descritos os conceitos de sistemas em tempo real, assim como, banco de dados em tempo real; na Seção 4 a arquitetura proposta, através da utilização em UML descrevendo os módulos que a mesma compõe bem como o funcionamento. Finalmente na Seção 5, apresentamos as conclusões e trabalhos futuros.

\section{ASSITÊNCIA DOMICILAR À SAÚDE (HOMECARE)}

A Assistência Domiciliar à Saúde (ADS) vem apresentando um novo modelo de serviços da saúde. Embora exista há algum tempo, esse tipo de atenção à saúde vem sendo enfatizada, em especial nas últimas décadas. Muitos fatores têm contribuído para o crescimento dessa modalidade de atendimento, no qual a ênfase do tratamento tem como prioridade as doenças crônicas, como sendo à base do sistema de saúde, e não mais apenas o tratamento com os cuidados agudos.

A questão da diminuição de custos no sistema de saúde, principalmente em relação aos planos de saúde, com a hospitalização de seus pacientes, também é um forte fator que ajuda na implantação do atendimento em ambiente domiciliar, que também oferece a privacidade e o conforto do atendimento ao paciente.

Algumas pesquisas indicam que através da ADS é possível não só diminuir a ocupação de leitos e a utilização de outros recursos hospitalares, consequentemente reduzindo custos, como também melhorar o tratamento do paciente (Dieckmann, 1994), (Kinsella, 2008), (Silva et al.; 2007) e (Mendes, 2001). Na ADS, em vez do paciente ir para o hospital, o profissional de saúde vai até a sua casa.

Um dos benefícios da ADS é a diminuição dos riscos de infecção, cujas taxas são altas devido à contaminação hospitalar. $\mathrm{O}$ uso da ADS faz com que as chances de infecção tendam a zero (Mendes, 2001). Esta característica é importante, dado que a infecção pode ser letal para pacientes com determinadas doenças, como por exemplo, a doença pulmonar obstrutiva crônica. Além da diminuição dos riscos de contaminação, através da ADS também é possível aumentar a autonomia e a privacidade do paciente. O aumento da autonomia do paciente e da família é promovido conforme a (o) enfermeira (o) orienta o paciente em seu tratamento, promovendo o autocuidado. $\mathrm{O}$ autocuidado representa a 
habilidade do paciente e da família em aprenderem a lidar com as sequelas da patologia básica e com o agravo, diminuindo assim, com o tempo, a necessidade dos serviços de profissionais de saúde.

\subsection{Atores de um Sistema Homecare}

Existem muitas entidades envolvidas em um sistema de homecare, as quais podem ser divididas da seguinte forma (Mcgee-lennon,2008):

- O (s) paciente (s): é o centro de um sistema de homecare. É possível que um único paciente esteja sob cuidados médicos para tratamento de mais de um problema. Também é possível que em um mesmo ambiente de homecare exista mais de um paciente que precisem de cuidados médicos como, por exemplo, um casal de idosos onde o marido tem problemas cardíacos e a esposa tem câncer;

- Os cuidadores: são definidos como as pessoas que fornecem a primeira assistência ao paciente, sejam médicos, enfermeiros ou familiares. Muitas vezes essa assistência é feita por um membro da família e isso pode se tornar bastante complexo quando o cuidador também precisa de cuidados, ou seja, quando um paciente depende da ajuda de outro paciente. Em situações como essa onde podem existir diferentes usuários finais, é necessário que o sistema se comporte de maneiras distintas de acordo com diferentes situações, ou seja, levando em consideração o contexto atual do ambiente.

- Visitantes: são os profissionais chamados para dar uma assistência. Neste grupo são incluídos, por exemplo, enfermeiras e paramédicos. Muitas vezes a diferença entre os visitantes e cuidadores não é bem definida. Se um profissional tem uma rotina de visitas ao paciente, de forma planejada, então ele é classificado como cuidador. Porém, se a visita ocorre de forma inesperada como em um atendimento a um paciente que está tendo um infarto, o profissional é classificado como visitante. Pessoas que entram no ambiente de homecare simplesmente para visitar o paciente também se encaixam neste grupo, desde que a visita não seja regular;

- Usuários remotos: neste grupo estão incluídos membros da equipe medica que acessam as informações do paciente mesmo sem se deslocar até a casa dele. Por muitas vezes um profissional pode está incluído neste grupo e também no grupo de visitantes.

- Fornecedores de tecnologia: são profissionais que projetam, desenvolvem e distribuem a tecnologia individual para o sistema de homecare e que podem impactar de alguma forma em como o sistema deve funcionar.

- Investidores institucionais: são pessoas que não são usuários diretos do sistema, mas que podem influenciar de alguma maneira em sua forma ou conteúdo. O ministério da saúde se encaixa neste grupo quando decide criar uma iniciativa para promover sistemas de homecare para um determinado propósito, por exemplo, para enfisema pulmonar.

- Demais investidores: pessoas que não usam diretamente o sistema, mas que tem algum tipo de interesse no mesmo. Neste grupo estão membros da família do paciente que não moram com o paciente, mas que tem interesse em seu estado de saúde, ou então que têm alguma obrigação financeira ou moral com relação a ele.

\subsection{Sistemas de Monitoramento Homecare}


O monitoramento de paciente homecare vem sendo muito utilizado atualmente, por ser uma prática de baixo custo, fácil implantação, acesso e aceitabilidade por parte de seus usuários. Em geral, esse monitoramento é realizado com pacientes portadores de doenças crônicas, sendo que nem sempre a relação médico- paciente é suficiente para garantir que o tratamento seja seguido de forma correta.

A modalidade, em geral, consiste em acompanhamento telefônico (direto ao paciente ou cuidador), priorizando o controle de uso de medicamentos controlados e informações sobre a situação do estado geral do paciente; e em visitas médicas e de equipes de enfermagem diretamente em domicílio, realizando consultas e os atendimentos básicos necessários.

Geralmente, as equipes de monitoramento são multidisciplinares, formadas principalmente por médicos, enfermeiros, fisioterapeutas, nutricionistas e psicólogos, que realizam visitas alternadas diretamente na residência do paciente, com o principal objetivo de prestar atendimento, verificando suas reais necessidades, indicando a melhor forma de tratamento e evitando a sua hospitalização.

Atualmente já existem diversas tecnologias disponíveis no mercado capazes de suportar aplicações voltadas para quem precisa ou prefere receber cuidados médicos em suas próprias casas. De acordo com (Mcgee-lennon,2008), os sistemas voltados para homecare devem possuir algumas características básicas, com por exemplo, xsensores que forneçam informações sobre o estado atual do paciente. O sistema deve levar em conta o contexto atual do ambiente em que o paciente se encontra como, por exemplo, sua localização na casa ou então que atividades estão realizando em determinado momento. Estes sensores também devem ser capazes de monitorar o estado de saúde do paciente, fornecendo informações de ordem fisiológica como temperatura, pressão sanguínea e batimentos cardíacos.

Em um sistema de homecare, qualquer pessoa que possa vir a utilizar o sistema de alguma forma deve ser considerada um usuário potencial. Desta forma, um sistema deve ser multiusuário. Neste grupo encontram-se os pacientes e os profissionais responsáveis por seus cuidados médicos. Os pacientes são vistos como fonte de informações e podem utilizar serviços do sistema para gerenciar informações personalizadas de sintomas e criar alertas de condições médicas. Por sua vez, os profissionais não usarão o sistema diretamente, como o paciente, mas de alguma maneira podem utilizar o sistema como forma de auxilio em seu trabalho.

Devido ao fato de ter grande parte do seu funcionamento baseado no contexto do ambiente, um sistema de homecare deve ter aplicações dinâmicas. Além da possibilidade de o estado de saúde do paciente pode mudar repentinamente, os outros usuários podem possuir diferentes desejos, necessidades e responsabilidade com o passar do tempo (Garde ,2006). Por exemplo, diferentes usuários podem preferir analisar os mesmos resultados de exames de formas diferentes, um através de texto e outro através de imagens. Um sistema desenvolvido com a possibilidade de atender as necessidades de cada usuário de forma individual resulta uma melhor qualidade do serviço e, consequentemente, maior aceitação do que um sistema com um único tipo de resposta para os usuários. Geralmente as pessoas responsáveis pelos cuidados do paciente, sejam familiares ou médicos, devem ter um conhecimento único das necessidades e preferencias do paciente, dessa forma deveriam ter o poder de gerenciar a forma como os serviços são executados, além de realizar mudanças de acordo com as novas necessidades do paciente. 
Ultimamente alguns trabalhos tem sido desenvolvidos em ambientes pervasivos com sistemas homecare, como (Carvalho et al.,2010), (Copetti, 2010), (Nogueira, et. al., 2012) e (Silva, et. al., 2013), desenvolveram aplicações para monitoramento de pacientes para auxiliar pessoas idosas que, apesar de não apresentarem um quadro clínico grave, necessitam de cuidados e atenção, como o Alzheimer, Esclerose Lateral Amiotrófica (ELA), Mal de Parkinson, dentre outras.

A quantidade de pessoas idosas, em especial aquelas com doenças crônicas ou condições críticas de saúde, tem crescido substancialmente nos últimos anos. Este cenário provoca uma alta demanda, exigindo cada vez mais da atual infraestrutura de serviços de saúde. A assistência domiciliar a saúde com o uso da tecnologia de computação pervasiva, pode representar uma solução.

Diante disso, muitas pesquisas estão sendo desenvolvida, entretanto, a ideia deste objeto de estudo é juntar conceitos, técnicas e tecnologias de forma diferenciada dos demais sistemas, combinando técnicas de banco de dados em tempo real (BDTR) com computação pervasiva que visa realizar o armazenamento, gerenciamento, processamento de informações úteis e em tempo hábil para quem de direito.

\section{SISTEMAS EM TEMPO REAL (STR)}

Os STR são sistemas que devem reagir a estímulos oriundos de um ambiente dentro de prazos específicos (Ramamritham et al., 2004) . Assim, para que uma tarefa em um STR seja executada com sucesso, ela deve ser executada dentro do intervalo de tempo determinado pelo sistema. Para isso, as tarefas de um STR devem incluir declarações explícitas sobre o tempo no qual o processamento deve ser realizado.

Aplicações e tarefas em tempo real são caracterizadas por restrições de tempo, ou prazos, que devem ser respeitados para obterem o comportamento temporal desejado ou necessário. Todas as tarefas de tempo real estão tipicamente sujeitas a prazos: os seus deadlines. A princípio, uma tarefa deve ser concluída antes de seu deadline.

Fazendo um paralelo, pode-se utilizar essa mesma definição para o ambiente hospitalar, onde, em uma alteração de quadro clinico é necessária à resposta ou tarefa adequada dentro de um espaço de tempo. Assim, aplicações e tarefas em tempo real são caracterizadas por restrições de tempo, ou prazos, que devem ser respeitados para obterem o comportamento temporal desejado ou necessário, os STR podem ser divididos em (Fernandes, 2005):

- Na Figura 1(A) é ilustrada uma tarefa com prazo estrito: uma tarefa tem prazo estrito quando qualquer resultado produzido após seu prazo final (deadline) é inútil para o sistema. Isso significa que qualquer tarefa estrita deve ser abortada quando não puder cumprir seu prazo, independentemente de sua consequência. Quando uma tarefa com prazo estrito perde seu prazo gera um valor negativo para o sistema e pode resultar em algo catastrófico, ou até mesmo perda de vidas humanas. Como por exemplo, o monitoramento de pacientes internados em Unidades de Terapia Intensiva (UTI), uma vez que, está relacionado com a saúde e eficiência no tratamento dos pacientes. É preciso notificar a ocorrência de anormalidades em tempo hábil pela equipe médica responsável para realizar as tomadas de decisões. 
- Na Figura 1 (B) é ilustrada uma tarefa com prazo firme: uma tarefa com prazo firme mesmo perdendo seu prazo final não gera nenhum efeito ou valor negativo para o sistema. Geralmente estas tarefas são reiniciadas quando perdem seu prazo, ou seja, não degrada o desempenho do sistema. Podemos citar como exemplo, depois da absorção do contraste pelo organismo do paciente ou após o decaimento do elemento radioativo, não há razão para terminar o exame. Será necessário outra administração de fármaco e novo exame.

- Na Figura 1 (C) é ilustrada uma tarefa com prazo suave: uma tarefa tem prazo suave quando o resultado produzido após seu prazo final sempre tem sempre algum valor, que vai perdendo sua utilidade à medida que se distancia do seu prazo final. Como exemplo, no ambiente hospitalar, temos serviço de laudagem, geração de imagem, medições de sinais vitais em monitorização de paciente em atendimento não urgente e estabilizado.

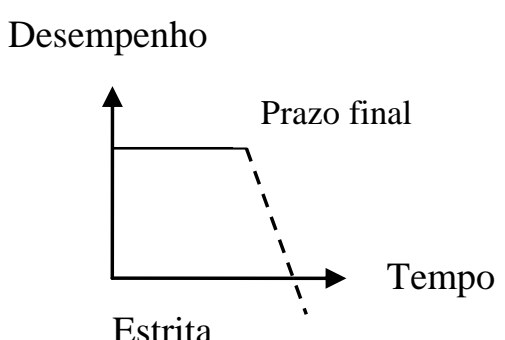

(A)
Desempenho

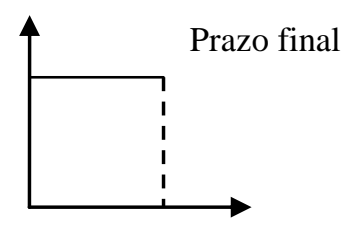

Firme

(B)
Desempenho

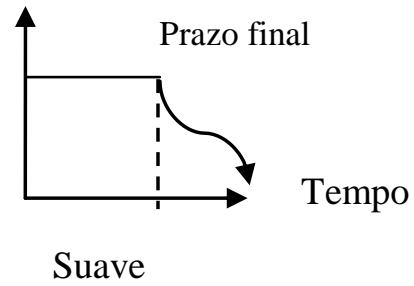

(C)

Figura 1 - Tarefas em Tempo Real

Outra característica temporal de tarefas em sistemas de tempo real está baseada na regularidade de suas ativações. Os modelos de tarefa comportam dois tipos de tarefas segundo suas ativações:

- Tarefas periódicas: quando as ativações de processamento de uma tarefa ocorrem, em uma sequência infinita, uma só ativação por intervalo regular chamando de período, essa tarefa é como periódica. Normalmente, essas tarefas iniciam no momento da inicialização do sistema e permanecem indefinidamente. Com exemplo, a leitura dos dados biomédicos do paciente e envio regularmente ao destinatário.

- Tarefas aperiódicas: quando a ativação do processamento de uma tarefa responde a eventos internos ou externos definindo uma característica aleatória nessas ativações, a tarefa é dita aperiódica. Como exemplo, podemos citar o recebimento de um alarme qualquer e envio deste com mais alta prioridade.

As tarefas periódicas, pela regularidade, e, portanto, pela previsibilidade, usualmente são associadas a prazos estritos, ou seja, são tarefas críticas. As tarefas aperiódicas pela falta de previsibilidade em suas ativações, normalmente, tem prazo suave associados a suas execuções, compondo, portanto, as tarefas brandas de um sistema em tempo real. Tarefas esporádicas que correspondem a um subconjunto das tarefas aperiódicas apresentam como característica central a restrição de um intervalo mínimo conhecido entre duas ativações 
consecutivas e por isso, podem ter atributos de tarefas críticas. As tarefas esporádicas, portanto são também associadas a prazos estritos (Shanker et al., 2008).

\subsection{Banco de Dados em Tempo Real (BDTR)}

Os BDTR são apropriados para gerenciar grandes volumes de dados compartilhados em aplicações de tempo real (Wang, 2012). Esses sistemas são projetados para, adicionadas às funcionalidades dos bancos de dados convencionais, incluírem as características necessárias para prover suporte à manipulação de dados e transações satisfazendo restrições temporais.

Os BDTR são úteis para STR com restrições de tempo não negociáveis, tais como aplicações redes de sensores, sistemas de monitoramento de pacientes, sistemas de monitoramento de satélites, onde os prazos temporais impostos aos dados e as transações não podem ser perdidos sob a pena de gerar algo grave que comprometa o sistema ou até mesmo a vida do paciente (Idoudi et al.; 2009).

Nas últimas décadas, o domínio de aplicação dos STR tem se expandido para aplicações que executam em ambientes imprevisíveis, tais como redes de sensores, comércio eletrônico, monitoramento de pacientes e bolsa de valores on-line, nos quais quanto mais prazos forem satisfeitos melhor é o desempenho do sistema (Ribeiro, 2006). Os ambientes de execução imprevisíveis e, consequentemente, é necessário de políticas de escalonamentos que possibilitem ao usuário especificar níveis de corretude e de desempenho aceitáveis para um BDTR.

Ultimamente algumas pesquisas sobre BDTR de modo a determinar métodos, técnicas e soluções para tratar com a complexidade imposta pela necessidade de garantir a integridade dos dados e satisfazer as restrições de tempo das transações têm sido realizadas (Lindstrom, 2008). Assim, novas abordagens têm sido introduzidas no escopo de BDTR com o objetivo de tratar com os diversos problemas decorrentes dos requisitos impostos, dentre os quais destacamos (Lindstrom, 2003), (Ramamritham, 2004), (Shanker, 2008), (Wang, 2012): Métodos para modelagem, gerenciadores de banco de dados, protocolos de controle de concorrência, linguagens de consultas.

A importância funcional dos BDTR se dá pelo fato destes suportarem as características de um banco de dados (BD), ou seja, de processar transações e garantir a consistência lógica dos dados e transações, como também as características de um sistema em tempo real (STR), de satisfazer às restrições de tempo impostas às tarefas (Shanke et al., 2008), como ilustrado na Figura 2. 


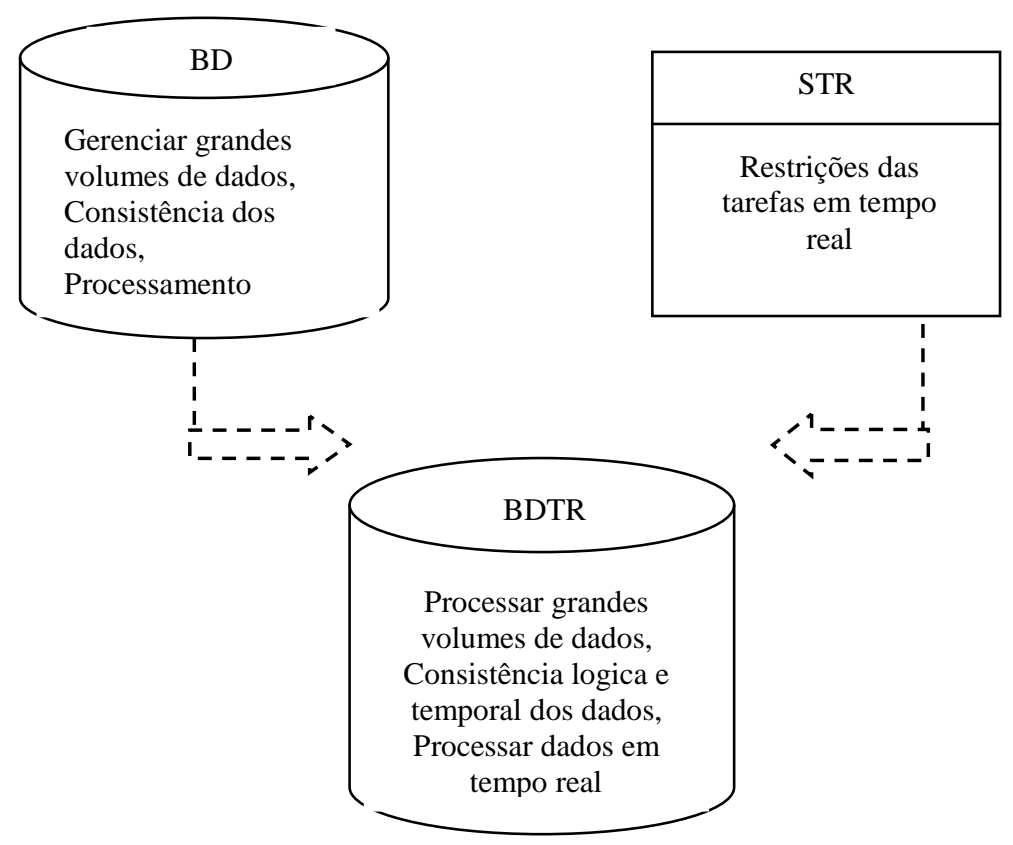

Figura 2 - Banco de Dados em Tempo Real

\section{ESPECIFICAÇÃO}

\subsection{Linguagem de modelagem unificada (UML)}

A UML (Unified Modeling Language) (Bezerra, 2006) é uma linguagem orientada ao paradigma de programação orientada a objetos e apesar dessa linguagem não ter sido desenvolvida especialmente para o paradigma dos sistemas de tempo real, vários estudos foram realizados para a sua adequação. É através de extensões da linguagem UML que é possível modelar sistemas com restrições temporais.

Sendo foi desenvolvida na década de 90 para homogeneizar as notações utilizadas pelas metodologias e ferramentas da modelagem da época. A UML tem a semântica rigorosamente definida, por meio de um metamodelo, e permite a modelagem tanto dos aspectos estruturais (estáticos) como aspectos comportamentais (dinâmicos) de um sistema.

A grande importância da modelagem é o fato de diminuir custos na medida em que erros podem ser eliminados ainda na fase de projeto do sistema. Para modelagem de sistemas de tempo real, é necessário modelar a infra-estrutura física do sistema, como processador e redes de comunicação e o relacionamento destes com o software. Também é preciso modelar o comportamento do sistema que tem como fundamento o acontecimento de eventos.

\section{MONITORAMENTO DE PACIENTES HOMECARE UTILIZANDO BANCO DE DADOS EM TEMPO REAL (MPH-BDTR)}

Nos hospitais, grande parte dos sistemas de monitoramento de pacientes faz uso de monitores multiparamétricos que apresentam algumas limitações no que diz respeito ao 
envio/disponibilização desses sinais vitais, integração com outros sistemas, assim como armazenamento dos dados por um determinado instante de tempo.

Em sistemas de monitoramento, podemos observar que, os pacientes principalmente em unidade de terapia intensiva (UTI) necessitam de acompanhamento contínuo dos seus sinais vitais, visando à detecção antecipada de situações de risco, permitindo a intervenção em tempo hábil pelos profissionais de saúde (Leite, 2011). Assim, ressalta-se a importância da equipe médica receber informações sobre os sinais vitais de um paciente sempre que for detectada uma possível situação de risco. Alguns trabalhos fazem uso de dispositivos móveis (Araújo, 2010). Esses dispositivos, notadamente os smartphones, estão cada vez mais presentes em nosso cotidiano. Associando esse fator a sua capacidade de integração com outras tecnologias, além do seu poder de processamento, demonstra que a utilização desses aparelhos apresenta-se como um componente interessante que pode ser acoplado aos diversos sistemas de monitoramento de pacientes.

No entanto, o monitoramento de pacientes em ambiente domiciliar tem impacto direto sobre as ações de monitoramento do paciente pelo sistema. Sendo assim, alguns trabalhos como (Copetti, 2010), (Silva, et. al., 2013) consideraram importante a medição de algumas variáveis desses ambientes, como temperatura e umidade, além também de identificarem a presença do paciente nos diversos ambientes da casa. Essa prática é mais comum em sistemas de monitoramento domiciliar para idosos, ou algum tipo de doença crônica.

Ultimamente observa-se um aumento progressivo do índice de hospitalizações após os 60 anos de idade, onde a ocorrência de reinternações é cinco vezes maior do que na faixa etária de 15 a 59 anos (Costa, et. al, 2000). Esse serviço não é oferecido exclusivamente ao publico idoso (acima de 60 anos). Entretanto, devido à prevalência de doenças crônicas nesta faixa etária, a dificuldade de locomoção, e com o aumento da expectativa de vida, o idoso é o público mais propenso à utilização da ADS ou homecare. Considerando que o custo de hospitalização por habitante do Sistema Único de Saúde (SUS) é mais alto para esta faixa etária, temos um panorama geral do atual problema de internação no país. A consequência direta do aumento do índice de hospitalizações é a superlotação das unidades, implicando em custos e na perda de qualidade do tratamento, principalmente em relação aos idosos com doenças crônico-degenerativas (diabetes e doenças cardíacas).

Um dos benefícios da ADS é a diminuição dos riscos de infecção, cujas taxas são altas devido à contaminação hospitalar. O uso da ADS faz com que as chances de infecção tendam a zero (Mendes, 2001). Além da diminuição dos riscos de contaminação, através da ADS também é possível aumentar a autonomia e a privacidade do paciente. O aumento da autonomia do paciente e da família é promovido conforme a (o) enfermeira (o) orienta o paciente em seu tratamento, promovendo o autocuidado. $\mathrm{O}$ autocuidado representa a habilidade do paciente e da família em aprenderem a lidar com as sequelas da patologia básica e com o agravo, diminuindo assim, com o tempo, a necessidade dos serviços de profissionais de saúde. O monitoramento dos dados do paciente, feitas de maneira autônoma em seu ambiente domiciliar pode, inclusive, gerar uma maior confiabilidade nos dados, uma vez solucionados os problemas de precisão dos dispositivos.

Dessa forma, este cenário gera uma alta demanda, exigindo cada vez mais de uma infraestrutura de serviços de saúde, para isso é preciso de ferramentas e técnicas que preservem a integridade do paciente sendo necessário de mecanismo eficiente de acesso 
aos dados. A melhor forma de realizar essa operação é o acesso em tempo real, possibilitando uma maior segurança e robustez à leitura das informações.

Assim, existe a necessidade de uma arquitetura de monitoramento de pacientes homecare utilizando BDTR e computação pervasiva, isso se deve ao fato do aumento substancial nos últimos anos de pessoas idosas, em especial aquelas com doenças crônicas ou condições criticas de saúde, como por exemplo: Mal de Parkinson, Esclerose múltipla, Doença de Huntington, Doença de Pick, Alzheimer.

\subsection{Arquitetura do Sistema}

A arquitetura consiste em sensores instalados na residência do paciente, onde o mesmo pode ser monitorado a todo o momento e em qualquer parte da casa. Dados fisiológicos (pressão arterial e frequência cardíaca), atividades realizadas pelo paciente (dormindo, andando, lendo) e condições do ambiente (temperatura e umidade) podem ser obtidos continuamente. Profissionais de saúde (por exemplo, cuidadores, enfermeiros, médicos) têm como acompanhar o paciente através de dispositivo móvel, computador servidor ou mesmo em suas residências, como apresentado na Figura 3. Os sensores utilizados pelo sistema fazem uso de tecnologias de baixo consumo de energia e não processam a informação coletada, caso seja necessário algum tipo de processamento antes do envio, o servidor local faz esse tratamento.

Localizado na casa do paciente temos um servidor local que está implantado em um sistema computacional sem restrição de bateria e com acesso constante a internet. $\mathrm{O}$ servidor local tem como objetivo garantir o envio continua das informações coletadas pelos sensores ao computador servidor, sendo, portanto, considerado uma ponte entre o sensor físico e o computador servidor. 


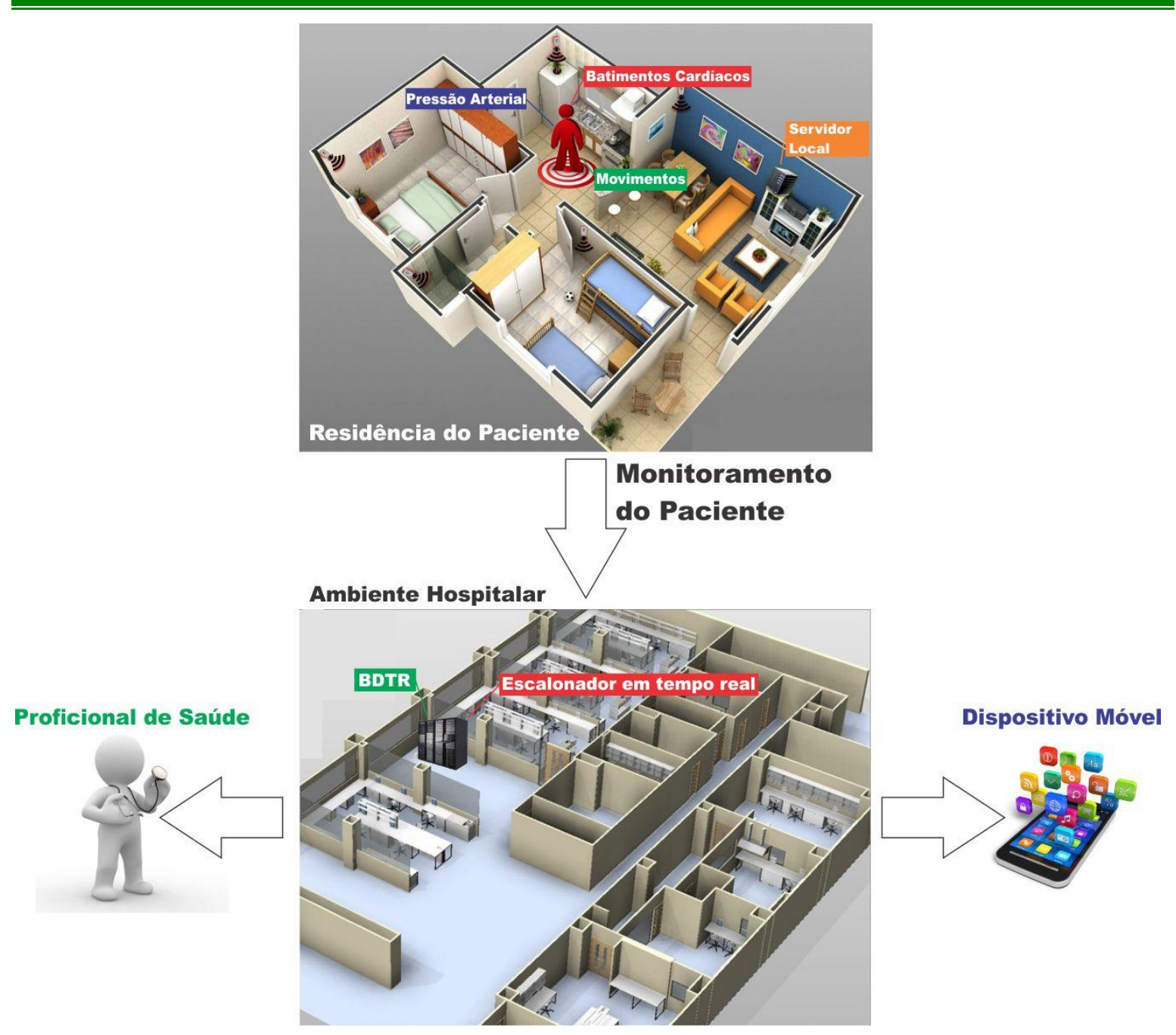

Figura 3 - Monitoramento de Paciente Homecare

Para modelagem da arquitetura do sistema, utilizamos a UML (Unified Modeling Language) (Bezerra, 2006) por permitir:

- Entender a estrutura dinâmica da organização na qual um sistema deve ser implantado;

- Assegurar que clientes, usuarios e desenvolvedores tenham um entendimento comum da organização;

- Apresentar um comportamento visual do sistema.

Dessa forma, descrevemos algumas funções da arquitetura do sistema, através do Diagrama de Caso de Uso. Na Figura 4 é mostrado um cenário da arquitetura proposta onde apresenta as seguintes funções: medição, coleta, análise de sinais vitais, recomendações terapêuticas e alarme que são monitoradas pelo sistema homecare e enviados a um servidor local. 


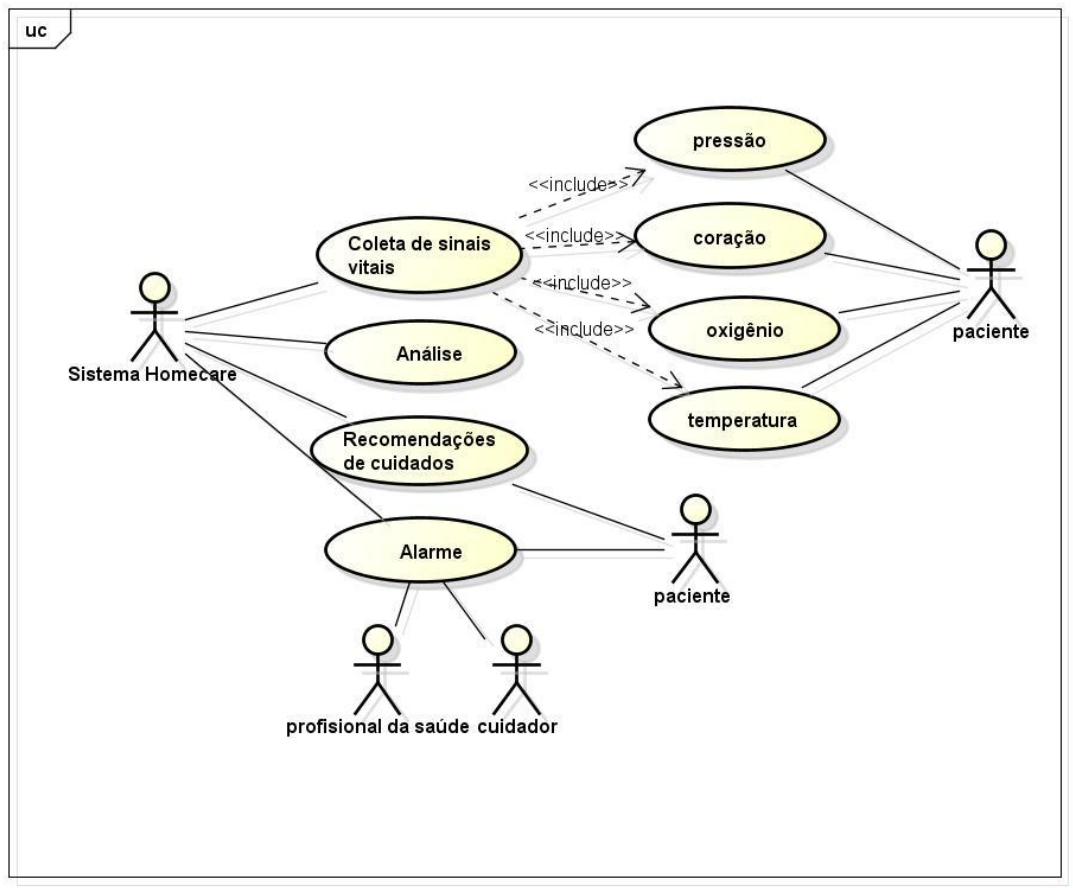

Figura 4 - Diagrama de Caso de Uso (Funções do sistema)

O servidor local possui o módulo controle de admissão, que obtém os dados fisiológicos e atividades do paciente, como podemos observar através do Diagrama de atividades na Figura 5.

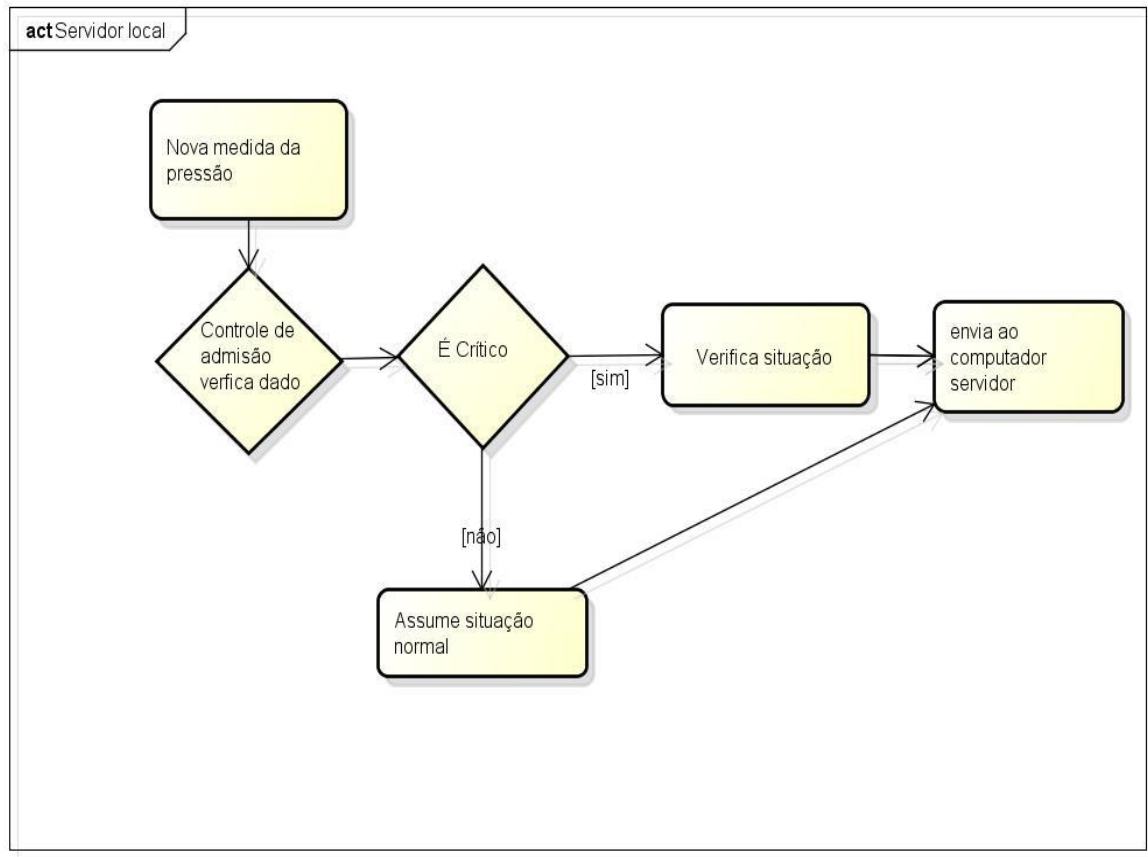

Figura 5 - Diagrama de Atividades (Servidor Local)

O computador servidor através do módulo BDTR faz a análise dos prazos das tarefas através do e scalonador em tempo real, enviando um alerta em caso de qualquer 
anormalidade para os profissionais de saúde e dispositivo móvel, como descrito pelo Diagrama de Atividades, mostrado na Figura 6.

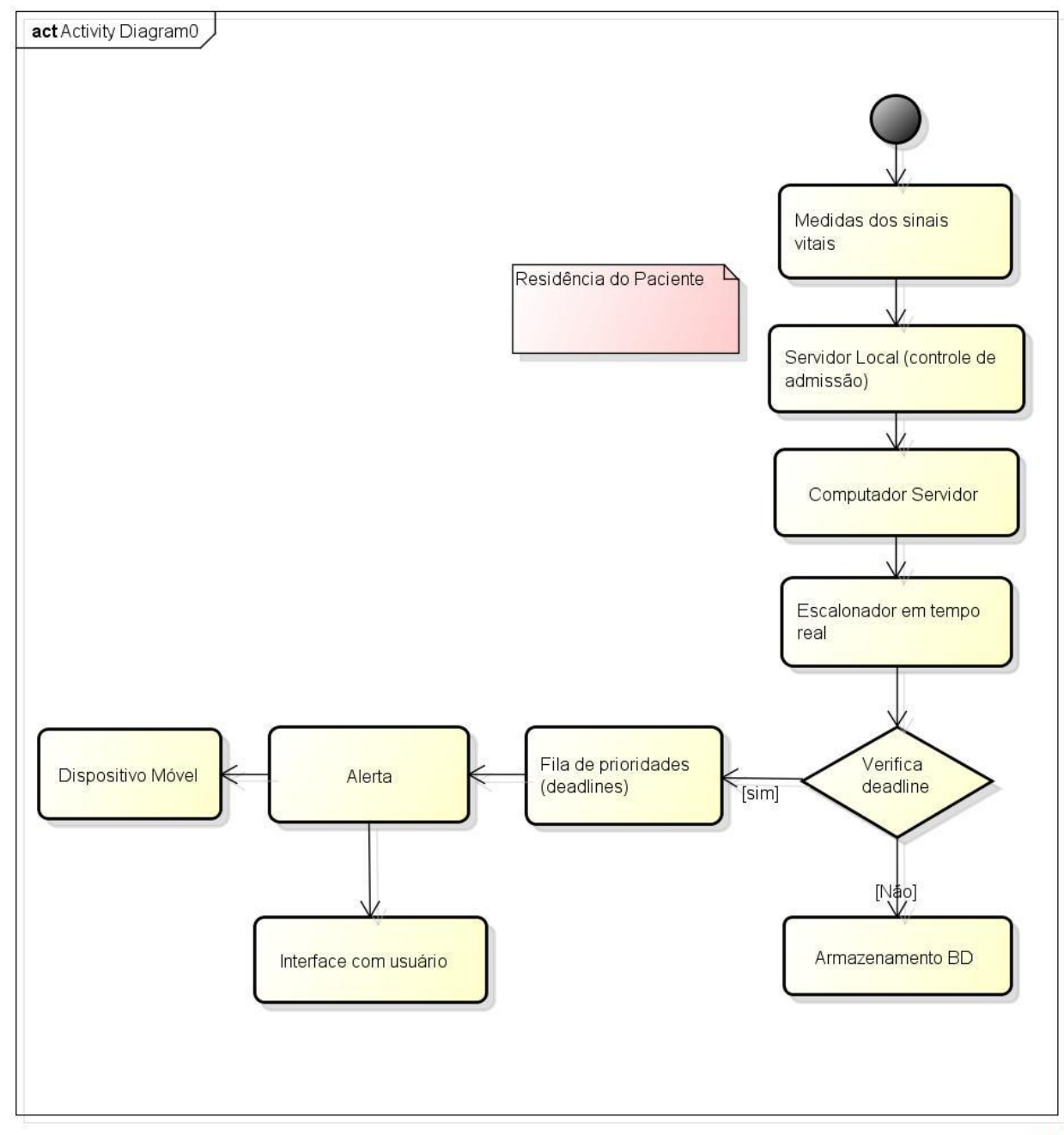

Figura 6 - Diagrama de Atividades (Computador Servidor)

O escalonador em tempo real mantém uma lista com todas as anormalidades recebidas para garantir as restrições de tempo dos dados recebidos. Assim, visando garantir que o atendimento que as mesmas ocorram dentro dos prazos impostos pelas restrições temporais (corretude temporal). A ordem de execução das anormalidades (tarefas) na lista depende de suas respectivas restrições de tempo.

Dessa forma, é atribuida para cada anormalidade de menor restrição temporal (deadline) a maior prioridade, colocando a mesma no topo da lista, sempre que uma nova anormalidade é recebida o escalonador reordena a lista.

As tarefas apresentam o seguinte comportamento temporal:

- Tempo de computação: o tempo e computação de uma tarefa é o tempo necessário para a execução completa da tarefa.

- Tempo de término: é o instante de tempo em que se completa a execução da tarefa. 
- Tempo de chegada: o tempo de chegada de uma tarefa é o instante em que o escalonador toma conhecimento de uma ativação dessa tarefa. Em tarefas periódicas, o tempo de chegada coincide sempre com inicio do período de ativação. As tarefas aperiódicas apresentam o tempo de chegada coincidindo com o tempo da requisição do processamento aperiódico.

- Tempo de liberação: o tempo de liberação de uma tarefa coincide com o instante de sua inclusão na fila de pronto (fila de tarefas prontas) para executar.

Assim, quando uma determinada tarefa é recebida pelo escalonador os parâmetros temporais e lógicos serão avaliados e deste modo, passando do estado tempo de chegada para tempo de liberação. Se a anormalidade tiver sido atendida antes do seu prazo final, a mesma será armazenada e disponibilizada para consultas e relatórios. Se a anormalidade não tiver sido atendida a mesma será abortada, como podemos observar através do Diagrama de Atividades na Figura 7.

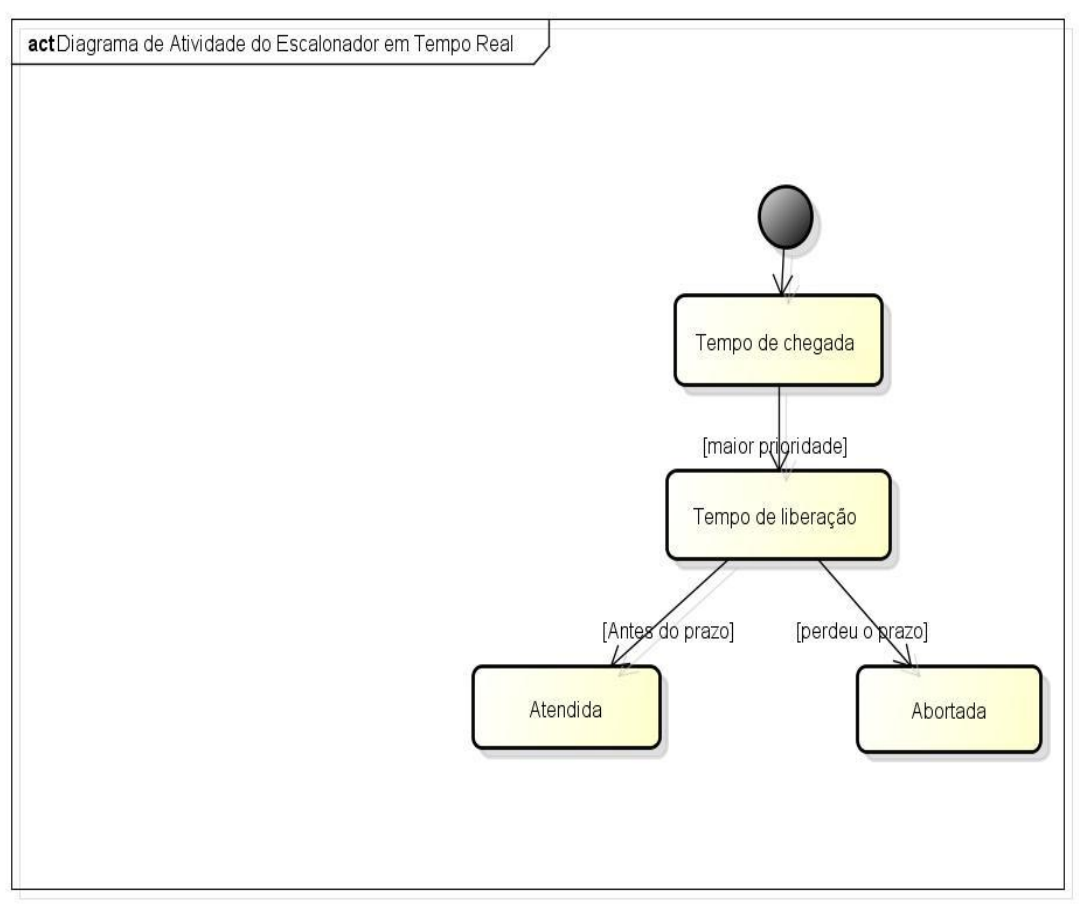

Figura 7- Escalonador em Tempo Real

Existem três tipos de consultas: (1) consulta de dados históricos: estas são consultas agregadas de dados históricos obtidos da rede; (2) consultas instantâneas: consultas na rede em um dado instante de tempo e; (3) consultas Longas: consultas na rede em um dado intervalo de tempo.

Após o cadastramento, qualquer alteração da normalidade do paciente será enviado para o médico através de e-mail, SMS. Uma consulta periódica é configurada no momento em que o paciente começa a ser monitorado. 


\section{CONCLUSÃO}

O desenvolvimento de aplicações voltadas à homecare aparece como um incentivo para que mais pessoas decidam pelo tratamento clínico diretamente em suas casas. Este tipo de serviço garante um maior conforto aos pacientes, uma vez que estarão em suas casas, além da possibilidade de ficar mais tempo com seus entes queridos. Com a popularização dessa forma de tratamento, as filas de pacientes em hospitais tendem a diminuir e, consequentemente, o serviço disponibilizado por estas instituições tende a melhorar consideravelmente.

Assim, essa pesquisa foi motivada principalmente devido à escassez de aplicações computacionais no tratamento de pacientes que recebem cuidados médicos diretamente em casa. Mesmo com a popularização de dispositivos computacionais, ainda não existem aplicações com BDTR e computação pervasiva capazes de interagir automaticamente com o usuário levando em consideração o contexto em que se encontram. O objetivo dessa pesquisa é melhorar a qualidade de vida dos pacientes e agilizar o trabalho dos profissionais que tratam dos mesmos.

Dessa forma, apresentou uma arquitetura que utiliza a computação pervasiva e BDTR para ambientes homecare, para isso algumas funcionalidades do sistema foram modeladas em UML para mostrar o comportamento do sistema.

\section{REFERÊNCIAS BIBLIOGRÁFICAS}

Andrade, L.M.; Martins, E. C.; Caetano, J. A.; Soares, E.; Bezerra, E. P.. Atendimento humanizado nos serviços de emergência hospitalar na percepção do acompanhante. Revista Eletrônica de Enfermagem, [S.1.], v.1, p.157, 2009.

Araújo, G., B. Modelo Arquitetural de Comunicação para Monitoramento de Pacientes Baseado em Middleware, Computação Móvel e Ubíqua. Natal -RN. Dissertação Mestrado em Engenharia Elétrica e Computação. Universidade Federal do Rio Grande do Norte, 2010 .

Carvalho, S., A., M. Um Sistema de Monitoramento Remoto de Pacientes usando Rede sem Fio. Dissertação de Mestrado da Pós- Graduação em Ciência da Computação da Universidade Federal de Minas Gerais, 2005.

Carvalho, S.T.; Erthal, M.; Mareli, D.; Sztajnberg, A.; Copetti, A.; Loques, O. Monitoramento Remoto de Pacientes em Ambiente Domiciliar. In: XXVIII Simpósio Brasileiro de Redes de Computadores - Salão de Ferramentas (SBRC), Gramado, RS, Brasil, 2010.

Copetti, A.. Monitoramento Inteligente e Sensível ao Contexto na Assistência Domiciliar Telemonitorada. Tese de Doutorado. IC-UFF, 2010. Orientador: J.C.B. Leite. Coorientador: O. Loques.

Costa, M.F.; Guerra, H.L.; Barreto, S.M. Diagnóstico da situação de saúde da população idosa brasileira: um estudo da mortalidade e das internações hospitalares públicas. Inf. Epidemiol. Sus, 9(1):43-50, 2000. 
Dieckmann, J.L. Home health administration: an overview. Handbook of home health care administration, pp. 3-13, 1994.

Fernandes, Y.Y.M. P. Técnica de Controle de Concorrência Semântico para Sistemas de Gerenciamento de Bancos de Dados em Tempo-Real. Dissertação de Mestrado. Universidade Federal de Campina Grande, 2005.

Garde, S.; Knaup, P. Requirements engineering in health care: the example of chemotherapy planning in paediatric oncology. Requir. Eng., Secaucus, NJ, USA, v.11, p.265-278, August 2006.

Idoudi, N.; Duvallet, C.; Bouaziz, R.; Sadeg, B. How to model a real-time database. UFR des Sciences et Techniques, 2009.

Kinsella, A. The home telehealth primer. Retrieved October, 10:2008, 2008.

Leite, C. R. M. Arquitetura Inteligente Fuzzy para Monitoramento de Sinais Vitais de Pacientes: Um Estudo de Caso em UTI. Tese de Doutorado pelo Programa de PósGraduação em Engenharia Elétrica e Computação - Universidade Federal do Rio Grande do Norte (UFRN)- 2011.

Marani, R., Perri, G.A. Design Of Advanced Electronic Biomedical Systems. International Journal of Advances in Engineering \& Technology, July 2012.

Mcgee-Lennon, M.R. Requirements Engineering for Homecare Technology. In: Proceeding of The Twenty-Sixth Annual Sigchi Conference on Human Factors in computing Systems, 2008, New York, NY, USA. Anais. ACM,2008. p.1439-1442.

Mendes, W. Homecare: uma modalidade de assistência à saúde. UnATI, UERJ, 2001.

Nogueira, C., Almeida, H., Perkusich, A., Arcabouço para o Desenvolvimento de Aplicações de Monitoramento Remoto e Auxílio de Pessoas com Doença de Alzheimer. SBCUP - IV Simpósio Brasileiro de Computação Ubíqua e Pervasiva, 2012. Curitiba -PR.

Lindstrom, J. Optimistic Concurrency Control Methods for Real-Time Database. Tese (Doutorado) - Department of Computer Science, University of Helsinki Finland, January 2003.

Ramamritham, K.; Son, S. and L. DiPippo. Real-Time Databases and Data Services. RealTime Systems, 28:179-215, 2004.

Ribeiro, N. P. Mecanismos de Qualidade de Serviços para o Gerenciamento de Dados e Transações em Tempo-Real. Tese de Doutorado. Universidade Federal de Campina Grande, 2006.

Silva, G.H.S; Morais, A. H.F; Vatentim, R.A.M; Dantas, R. R; Cavalcanti, L. R.C; Junior, M.E.T.J. Um anjo para ELA: arquitetura baseada em visão computacional aplicada ao monitoramento on-line de parâmetros respiratórios de pacientes com Esclerose Lateral Amiotrófica (ELA) em ambiente hospitalar. Revista Brasileira de Engenharia Biomédica, Volume 29, Número 2, p. 206-210, 2013. 
Silva, L.; Galera, S.A.F.; Moreno, V. Encontrando-se em casa: uma proposta de atendimento domiciliar para famílias de idosos dependentes. Acta Paul Enferm, 20(4):397403, 2007.

Shanker, U., Misra, M., Sarje, A.K. Distributed real time database systems: background and literature review. Distrib Parallel Databases (2008) 23: 127-149. Springer Science.

Zarghami, A.; Eslami, M.Z.; Sapkota, B.; Sinderen, M. Toward Dynamic Service Provisioning in The Homecare Domain. In: Pervasive Computing Technologies for Healthcare, $20115^{\mathrm{TH}}$ Internacional Conference On, 2011. Anais, p 292-299.

Wang, J., Han, S., Lam, K., Mok, K. A. Maintaining data temporal consistency in distributed real-time systems. Springer Science Business Media, LLC 2012. 